\title{
Ueber den Reizwerth sinusoidaler Ströme von hoher Frequenz.
}

Von

J. K. A. Wertheim Salomonson (Amsterdam).

(Mit 4 Textfiguren.)

Nachdem von Bernstein ${ }^{\mathbf{1}}$ ) gezeigt worden war, dass Reizung eines Froschischiadicus mit etwa 800 Reizen pro Secunde bei genügender Intensität noch einen glatten Tetanus liefern konnte, baben verschiedene Forscher dasselbe erreichen können bei weit höheren Frequenzen. So wurde mit Bestimmtheit von $\mathrm{Kronecker}{ }^{2}$ ) nachgewiesen, dass mit den dem von ihm construirten magnetischen Toninductorium entnommenen Strömen mit einer Frequenz von etwa 20000 pro Secunde noch immer ein Tetanus erhältlich war.

Während $\operatorname{Roth}^{3}$ ) vergeblich versucht hatte, einen Eindruck über die Intensität der von ihm angewandten Reize hoher Frequenz - bis etwa 5000 pro Secunde - zu gewinnen, gelang es v. Kries ${ }^{4}$ ) mittelst einer sehr interessanten Methode, auch den relativen Reizwerth solcher Ströme zu bestimmen. Sein Instrumentarium gestattete ihm aber nicht, erheblich höhere Frequenzen zu erreichen, als seine Vorgänger schon benutzt hatten; bis jetzt scheint K ronecker die höchste Frequenz erreicht zu haben. Kronecker hat aber, soviel mir bekannt ist, keine vergleichenden Bestimmungen der Reiz-

1) Bernstein, Untersuchungen über den Erregungsvorgang im Nerven- und Muskelsysteme. Heidelberg 1871.

2) Kronecker und Stirling, Die Genesis des Tetanus. Du BoisReymond's Arch. 1878 S. 38. Daselbst S. 27 u. f. auch die ältere, hier nicht angefübrte Literatur.

3) Roth, Ueber die Wirkung höchster Reizfrequenzen anf Muskeln und Nerven. Pflüger's Arch. Bd. 42 S. 91-103.

4) v. Kries, Ueber die Erregung des motorischen Nerven durch Wechselströme. Berichte d. Verhandl. d. Naturf. Gesellsch. i. Freiburg i. Br. Bd. 8 S. $170-206.1885$. 
werthe versucht oder wenigstens veröffentlicht. Jedenfalls eignet das Toninductorium sich auch nicht sehr zu derartigen Bestimmungen, obgleich es ein ausserordentlich bequemes, zuverlässiges und einfaches Instrument darstellt.

Vor Kurzem hat Einth ove $n^{1}$ ) mit Bestimmtheit nacbgewiesen, dass ausserordentlich schnelle gedämpfte Wechselströme einen wirksamen Reiz für Muskeln und Nerven darstellen: Frequenzen bis etwa $10^{6}$ pro Secunde erzeugen regelmässig Zuckungen. Einthoven hat zu gleicher Zeit bewiesen, dass derartig frequente Ströme in die Halbleiter eindringen können und durchaus nicht an der Oberfläche derselben verlaufen müssen, wie von Manchem irrthümlicher Weise per analogiam mit dem Verhalten der gleichen Ströme Eisen und Kupfer gegenüber angenommen wurde.

Ueber das Verhalten ungedämpfter sinusoidaler Ströme von einer Frequenz über 20000 (Kronecker) pro Secunde war bis jetzt nichts bekannt.

Zweck dieser Arbeit ist, diese Lücke theilweise auszufüllen und zu gleicher Zeit Einiges mitzutheilen über den Reizwerth derartiger Ströme.

\section{Methodik.}

Das Princip der von mir verwendeten Methode zur Hervorbringung von sehr frequenten sinusoidalen Strömen rührt von Dudde l1 ${ }^{2}$ ) (1900) her. Wird ein galvanischer Lichtbogen mittelst eines Condensators und eines in Serie geschalteten induciven Widerstandes kurz geschlossen, dann giebt der Lichtbogen einen pfeifenden Ton. Dabei geht durch den Condensatorkreis ein Wechselstrom, dessen Frequenz übereinstimmt mit der Tonhöhẻ des pfeifenden Tones. Durch passende Aenderung der Condensatorcapacität, des Selbstinductionscoëfficienten, der Lichtbogenspannung (D u d d ell) und der Stromstärke [Wertheim Salomonson ${ }^{3}$ )] lässt sich die

1) Einthoven, Ueber Nervenreizung durch frequente Wechselströme. Pflüger's Arch. Bd. 82 S. 101-138.

2) Duddell, Or rapid variations in the current through the direct-current arc. The Electrician vol. 46 p. $269-273$ u. $310-313.1900$.

3) Wertheim Salomonson, Tijdschrift v. Geneeskunde 1902 Deel 1 p. 967-972. Verslag der Vereeniging voor Electrotherapie en Radiologie. Compt. rend. du deuxième Congrès International de Radiologie et d'Électrologie Médicales à Bern 1902 p. 219. - Stroomsterkte en toonhoogte bij den"fluitenden. 
Frequenz des alternirenden Stromes auf jeden beliebigen Werth bringen.

Diese Ströme können wie alle Wechselströme transformirt werden und somit auf die gewunschte, zur Muskel- oder Nervenerregung nöthige Spannung und Intensität herabgesetzt oder erböht werden.

\section{Instrumentarium.}

Die von mir verwendete Bogenlampe war eine kleine Gleichstrom-Nebenschlusslampe von Körting und Matthiessen, welche bei 2-4 Ampère ein ruhiges Licht lieferte. Die Spannung lässt sich in weiten Grenzen beliebig ändern durch Verstellung der Regulirschraube. Die günstigste Spannung für unseren Zweck ist n i e d rig e r, als für ein ruhiges Brennen zu Beleuchtungszwecken erforderlich ist, und beträgt etwa 36-42 Volt; um eine niedrige Wechselzahl in dem Condensatorkreis zu erhalten, nimmt man die höhere Spannung, bei höheren Wechselzahlen nimmt man die Spannung immer niedriger, bis zu 36 Volt. Die günstigste Ampèrezahi beträgt etwa 2,6 bis 2,8 Ampère.

Die Spannung der Elektricitätsquelle soll wo möglich 110 Volt oder mehr betragen. Eine Accumulatorbatterie ist einer Dynamomaschine vorzuziehen. Die Stromstärke wird durch einen Vorsehaltwiderstand auf das richtige Maass gebracht. Als Kohlen dürfen nur homogene Kohlen verwendet werden, und zwar an beiden Polen. Ich gebrauchte meistens Kohlen von 7-9 mm Durchmesser.

Der. Gebrauch einer Hauptstrom- oder Differentiallampe ist weniger $\mathrm{zu}$ empfehlen. Noch weniger eignet sich ein Handregulator für unseren Zweck. Auch eine bei Abschluss der Luft in Stickstoff brennende Bogenlampe ist weniger zu empfehlen. In den Hauptstromkreis wird vortheilhafter Weise noch eine Drosselspule eingeschaltet.

Der Condensatorstromkreis enthält erstens einen Condensator mit regulirbarer Capacität.

lichtboog. Kon. Akad. v. Wetensch. te Amsterdam p. 381. 5. Nov. 1902. Measurement of the frequency of very rapidly alternating Currents. The Electrician $13^{\text {th }}$ Nov, 1903. - Courants de haute fréquence non-amortis. Arch. d'Électricité Médicale Sept. 1903. Association franç. p. l'avancement d. Sciences. Congrès d'Angers. 4-11 Août 1903. 
Ich verfügte über:

einen zwölftheiligen Präcisionscondensator von Siemens \& Halske von $0,001-1$ M.-F.,

einen. zwölftheiligen Präcisionscondensator von. Edelmann von 0,001-1,11 M.-F., einen Papiercondensator von Desruelles von 1,0 M.-F., einen achttheiligen Micacondensator von 0,01-1,5 M.-F.

Zusammen also etwa 4,5 M.-F., bei Parallelstellung sämmtlicher Condensatoren.

Ausserdem enthält dieser Condensatorkreis eine Selbstinduction. Dieselbe bildete die primäre Spirale eines Schlittenapparats und bestand aus 180 Windungen eines $1^{3 / 4} \mathrm{~mm}$ dicken Drahts oder auch aus 36 Windungen eines $3 \mathrm{~mm}$ dicken Drahts. Ein Eisenkern war nicht nur überflüssig, sondern sogar schädlich. Wie fein der Eisenkern auch verteilt sein mag, er erwärmt sich doch in wenigen Secunden. Als ich nach dem Beispiele Braun's ${ }^{1}$ ) Ferrum Hydrogenio reductum in einer Glasröhre mit Paraffinöl als Kern benutzte, beeinträchtigte auch dieser die Wirkung erheblich.

Zuletzt war meistens noch ein Hitzdrahtampèremeter, von 0 bis 10 Ampère anzeigend, von Hartmann \& Braun eingeschaltet, das anfangs zu Messzwecken, späterhin nur als Stromzeiger verwendet wurde.

Wir werden diesen Condensatorstromkreis späterhin als den primärèn bezeichnen.

Ueber die primäre Rolle des in den primären Condensatorkreis geschalteten Inductionsapparats kann eine secundäre Rolle geschoben werden, welche uns wieder Wechselströme liefert, welche für Reizzwecke verwendet werden können. Da unser Inductionsapparat ein „Lufttransformator" ist, werden die primären Ströme ohne Difformation transformirt.

Von secundären Rollen besass ich zwei verschiedene, und zwar eine Rolle mit zwei Lagen von je 225 Windungen dünnen Drahts - $0,3 \mathrm{~mm}$ Durchmesser —, welche entweder jede für sich oder beide hinter einander geschaltet oder parallel geschaltet gebraucht werden konnten; eine zweite Rölle trug 72 Windungen eines $0,9 \mathrm{~mm}$ dicken Drahts in einer Lage.

1) Braun, Drude's Annalen 1903. 


\section{Bestimmung der Frequenz.}

Man würde meinen, dass die Frequenz des Wechselstroms bloss abhängig wäre von der Selbstinduction $L$ und der Capacität $C$ in primären Condensatorkreis, narh der bekannten Formel $T=2 \pi \sqrt{L C}$, wo $T$ die Dauer einer completten Oscillation angiebt. Es wurde dies zuerst von $\mathrm{Duddel} 1^{1}$ ) angenommen und auch von Jane $\mathrm{t}^{2}$ ) als feststehend betrachtet; dieser meinte daher eine Methode zur Bestimmung von $L$ hierauf gründen zu können. Von mir selbst und

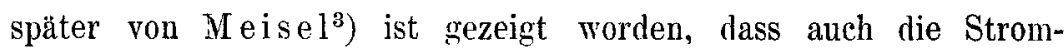
stärke des Lichtbogens und dessen Spannung von Einfluss sind auf die Frequenz, so dass die obige Formel nicht angewendet werden darf.

A. Peukert ${ }^{4}$ hat indessen eine brauchbare Methode angegeben für Messung der Frequenz. Misst man die Spannung des Lichtbogens mit einem Hitzilraht-Voltmeter, wobei man $E_{1}$ bekommt, und mit einem Weston-Instrument, welches $E_{1 x}$ anzeigt, dann beträgt die mittlere Wechselstromspannung: $E=\sqrt{E_{1}{ }^{2}-E_{11}{ }^{2}}$.

Ist die Capacität des Condensators zu $C$ Farad und die Wechselstromstärke im Condensatorkreis mit einem Hitzdrahtampèremeter zu $J$ bestimmt, dann ist die Frequenz:

$$
n=\frac{J}{2 \pi C E} .
$$

Während diese Methode bei niedrigen Frequenzen sehr einfach und vollkommen zuverlässig ist, ist dies nicht mehr der Fall bei hoben Frequenzen. Die Bestimmung von $E$ aus zwei Voltmeterablesungen wird dann ungenau, da die beiden Werthe dann nur wenig verschieden sind. Ausserdem erheischt die Methode drei gleichzeitige Ablesungen von drei nicht immer ruhigen Instrumenten, wozu drei geübte Beobachter nöthig sind. Schliesslich lässt die Methode im Stich, wenn der Wechselstrom nicht ein rein sinusoidaler ist. Ich habe diese Methode später nicht mehr benutzt und nur noch die folgende Methode angewandt.

B. Eine ausserordentlich genaue und bequeme Methode, der $\operatorname{ich}^{5}$ )

1) Duddell, 1. c.

2) Janet, Compt. rend. Acad. d. Sc. 1902 Nr. 8 p. 15.

3) Meis el, Physik. Zeitschr. Bd. 4 S. 532.

4) Peukert, Elektrotechn. Zeitschr. 1901 S. 467.

5) Wertheim Salomonson, Measurement of the frequency of very rapidly alternating Currents. The Electrician 13 th Nov. 1903. 
den Namen Resonanzmethod e beilegen möchte, wird in folgender Weise ausgeführt.

Bringt man in der Nähe des primären Condensatorkreises einen zweiten, völlig von dem ersten getrennten Stromkreis an, der aber auch Selbstinduction und Capacität enthält, dann wird in diesem Stromkreis, den ich als secundären Condensatorstromkreis bezeichnen werde, die Elektricität in Oseillation gerathen, sobald die Dauer der dem secundären Condensatorkreis eigenen Oscillationen übereinstimmt mit der Frequenz im primären Condensatorkreis. Wir brauchen nur noch eine Vorrichtung, die anzeigt, ob die Elek-

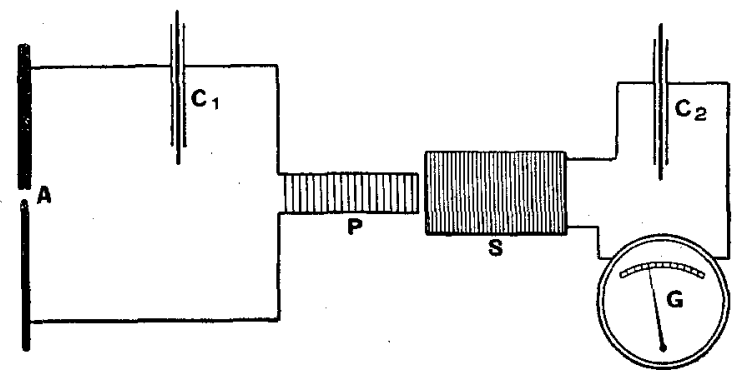

Fig. 1.

tricität im secundären Condensatorkreis oscillirt oder nicht. Hierzu verwende ich wieder ein Hitzdrahtampèremeter von Hartmann und Braun, von $0-0,3$ Ampère calibrirt. Schwingt der secundäre Condensatorkreis mit, dann entsteht ein Wechselstrom, der vom Hitzdrahtinstrument angezeigt wird. Man braucht jetzt nur die Capacität des secundären Condensatorkreises zu ändern, bis ein maximaler Ausschlag erhalten wird und jede Aenderung der Capacität - sei es Vergrösserung oder Verkleinerung - diesen Ausschlag herabsetzt. Ist diese Capacität bestimmt, danu ist complette Resonanz erreicht, und ist die eigene Schwingungsdauer des secundären Condensatorkreises genau abgestimmt auf die Frequenz der Oscillationen im primären Kreis.

Um jetzt die Frequenz zu berechnen, braucht man nur den Selbstinductionscoëfficienten $L_{11}$ und die Capacität $C_{11}$ im secundären Condensatorkreis zu. kennen, aus denen man nach der Formel $T=2 \pi \sqrt{L_{11} C_{11}}$ die gesuchte Frequenz berechnet. Man beachte, dass $L_{11}$ dabei in Henry und $C_{11}$ in Farad ausgedrückt werden soll, um $T$ in Secunden zu erhalten. $\frac{1}{T}=n$ ergiebt die Frequenz.

E. PfIüger, Archir für Physiologie. Bd. 106. 
Für die Capacität benutzte ich den schon erwähnten Condensatorsatz von Edelmann; die Selbstinduction wurde geliefert von den ebenfalls schon erwähnten zwei secundären Rollen des Inductionsapparates. Die Coëfficienten wurden mit der peinlichsten Sorgfalt nach drei versehiedenen Methoden bestimmt, zu:

$78,84 \cdot 10^{-6}$ Henry für Rolle I mit 72 Windungen, $664,0 \cdot 10^{-6}$ Henry $" \quad$ II, innere Drahtlage, $225 \mathrm{~W} .$,
$684,7 \cdot 10^{-6}$ Henry $" \quad$ II, äussere Drahtlage, $225 \mathrm{~W} .$,
$2698 \cdot 10^{-6}$ Henry $" \quad$ II, beide Drabtlagen in Serie, $450 \mathrm{~W}$.

Eine directe Vergleichung $z$ wischen Rolle I und der äussere Lage von Rolle II zeigte ein Grössenverhältniss von $8,72: 1$, während obige Zahlen 684,7:78,84 sich verhalten wie 8,69:1. Die Uebereinstimmung ist eine genügende.

Ich möchte hier noch hinzufügen, dass ich auch meine Condensatorsätze genau geaicht habe, wobei der Edelmann'sche Satz sich auf weniger als $0,2 \%$ genau erwies; nur die vier kleinsten Nummern zeigten etwas grössere Fehler, die immer noch unter $0,67 \%$ blieben. Der Siemens'sche Condensator war viel weniger genau und zeigte bei einer der grösseren Abtheilungen sogar einen Fehler von $4 \%$, während die vier kleinsten Abtheilungen fast $15 \%$ zu klein waren. Ueber die Methoden zur Bestimmung von Selbstinductionscoëfficienten und Capacitäten weise ich hin auf die Bücher Kohlrausch's, Fleming's u. s. w.

Der grösste Vortheil, den meine Resonatormethode bietet, liegt weniger in der exacten und ausserordentlich einfachen Bestimmung der Frequenz, sondern hauptsächlich in dem Umstande, dass sie eventuelle Schwingungen von einer anderen Schwingungsdauer, Obertöne, vollständig eliminirt ${ }^{1}$ ). Bestände der ursprüngliche Wechselstrom aus zwei sinusoidalen Schwingungen verschiedener Perioden, so wird in dem Resonator nur einer von diesen Componenten verstärkt, und zwar derjenige, dessen Periode mit der Schıingurısszahl des Resonators (also des secundären Condensatorkreises) übereinstimmt, während die andere Componente derartig geschwächt wird, dass diese Schwächung für praktische Zwecke einer vollständigen Flimination gleichkommt. Selbst wenn also der ursprünglich im

1) Obertöne treten fast nur bei grosser Gleichstromintensität auf, etwa bei fünf oder sechs Ampère. Bei geringer Stromstärke, unter vier Ampère, sind sie meist nicht in nachweisbarer Menge vorhanden. 
primären Condensatorkreis circulirende Wechselstrom ein zusammengesetzter sinusoidaler Strom wäre, so ist jedenfalls der Strom im secundären Condensatorkreis ein rein sinusoidaler.

C. Noch eine dritte Methode ist von mir angewandt worden, hauptsächlich zur Controle der zweiten Methode. Diese "photographische" Methode besteht darin, dass der Lichtbogen mittelst eines schnell rotirenden Spiegels auf einer beweglichen photographischen Platte photographirt wird. Der pfeifende Lichtbogen zeigt namentlich nicht eine constante Lichtintensität, sondern eine periodisch wechselnde: die Frequenz der Schwankungen der Lichtintensität ist nothwendig gleich der Frequenz der elektrischen

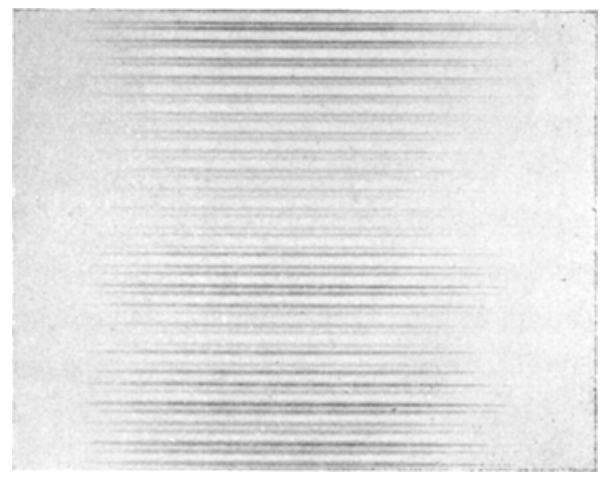

Fig. 2.

Schwingungen im primären Condensatorkreis, weil sie von diesen erzeugt werden. Der Ton, welcher von dem periodisch schwankenden Lichtbogen erregt wird, ist auch mit dieser Frequenz übereinstimmend. Die Ausführung der photographischen Fixirung dieser Lichtschwankungen geschah in der Weise, dass der Lichtbogen mittelst eines photographischen Objectivs auf einer Trockenplatte projectirt wurde, nachdem die Lichtstrahlen von einem kleinen Spiegel reflectirt waren. Der Spiegel konnte mittelst eines Motors bis $400 \mathrm{Mal}$ pro Secunde gedreht werden. Dabei wurde das Bild des Lichtbogens zu einem Bande ausgezogen. Da bei jeder Umdrehung des Spiegels das Lichtband auf die nämliclse Stelle der Platte fallen würde, musste die Platte inzwischen verschoben werden. Die Figur 2 giebt uns einen Abdruck des erhaltenen Negativs und zeigt eine Reihe von Lichtbändern, von denen die unteren deutliche Intermissionen erkennen lassen. Die Umdrehungsgeschwindig- 
keit wurde gemessen, indem jede 50. Umdrehung mittelst eines Pfeil'schen Signals gleichzeitio mit Secundenmarken auf ein Kymographion verzeichnet wurde.

Die experimentellen Daten des Versuches waren:

Lichtbogenspannung (West o n-Instrument) . 34,7 Volt

Stromstärke (Westo n-Instrument) . . . . 2,1 Ampère

Durchmesser der homogenen Kohlen. . . . 7 und $4 \mathrm{~mm}$

Primäre Selbstinduction $4 \mathrm{~mm}$ Draht . . 36 Windungen

,

Capacität . . . . . . . . . 0,104 Mikrofarad

Secundärer Selbstinductionscoëfficient . . . 78,84 $10^{-6}$ Henry

Capacität . . . . . . . . . $0,017 \cdot 10^{-6}$ Farad

Nach Methode B berechnete Frequenz . . 138000 pro Secunde

Abstand des Spiegels vom Lichtbogen . . . $29,4 \mathrm{~cm}$

Negativ gab ein verkleinertes Bild . . . . 0,579

Umdrehungsgeschwindigkeit des Spiegels . . 241,0 pro Secunde

Distanz der Lichtmaxima auf das Negativ . . $3,87 \mathrm{~mm}$

Nach Methode C berechnete Frequenz . . . 133200 pro Secunde.

Die Uebereinstimmung zwischen Methode $\mathrm{C}$ und "B ist eine genügende; im Weiteren ist ausschliesslich meine Resonatormethode zur Frequenzbestimmung benutzt.

Bevor wir zur Beschreibung der Versuche schreiten, möchte ich noch bemerken, dass der pfeifende Lichtbogen nur dann hörbar pfeift, solange die Frequenz unter etwa 43000 Schwingungen pro Secunde bleibt. Wird die Frequenz höher, so hört man keinen Ton mehr. Das Bestehen der Schwingungen verräth sich jedoch sogleich durch die. Wirkung des Stromes auf das thermische Ampèremeter oder durch die abstossende Wirkung auf einen benachbarten Leiter, z. B. einen Aluminiumring, oder endlich durch die physiologische Wirkung auf Muskeln oder Nerven. Die Ströme sind stark genug, um durch die intacte Haut des Menschen hindurch kräftige Contractionen zu erregen.

Mit dem oben beschriebenen Instrumentarium, das Wechselströme von rein sinusoidaler Form und beliebiger Frequenz liefern kann, habe ich eine Reihe von Reizversuchen an Froschpräparaten vorgenommen. Zweck dieser Versuche war die Bestimmung der geringsten Stromstärke, die gerade ausreichte, einen glatten Tetanus zu erzeugen, wenn die Frequenz in weiten Grenzen geändert wurde. Wir bedurften also eines Mittels zur genauen Messung dieser Ströme. 
Von vornherein wurde Abstand genommen von einer directen Messung der Stromstärke. Dies wäre nur ausführbar gewesen mit Instrumenten, welche eine hohe Selbstinduction besitzen, wie das Elektrodynamometer von Bellati-Giltay. Während bei den niedrigen Frequenzen hierbẹi keine Schwierigkeit bestehen würde, hätte die Anwendung bei hohen Frequenzen sehr grosse Schwierigkeit verursacht. Durch die grosse Selbstinduction des Messinstruments wären die Wechselströme so ausserordentlich geschwächt worden, dass keine Erregung des Nerven oder des Muskels mehr stattfinden würde. Wir hätten in diesem Fall die Selbstinduction compensiren müssen mittelst passender Condensatoren; die Beschaffung von genau calibrirten Condensatoren von passender Grösse hätte eben das grösste Hinderniss abgegeben, da die Grössenordnung dieser Condensatoren etwa $0,00001-0,001$ Mikrofarad betragen hätte. Ausserdem hätte man bei jedem Versuche zuerst die nöthige Condensatorgrösse berechnen müssen, wobei zu viel Zeit verloren gegangen wäre.

Ich habe mich darum auf die viel genauere directe Bestimmung der Potentialdifferenz beschränkt, die gerade genügt, einen Strom durch das Präparat zu schicken, welcher einen minimalen Tetanus erzeugt. Kennt man den Widerstand des Präparats, dann lässt sich die Stromstärke in einfachster Weise berechnen.

Die Bestimmung der elektromotorischen Kraft des Wechselstromes konnte in verschiedener Weise erfolgen.

1. Wenn in dem secundären Condensatorkreis die Capacität des Condensators $=C_{11}$ ist, die Stromstärke $i_{11}$ beträgt, bei einer Frequenz $n$, dann beträgt die Klemmenspannung des Condensators:

$$
E_{2}=\frac{i_{11}}{2 \pi n C_{11}^{\prime}}
$$

Der Versuch lässt sich ausführen, indem man bei einem passenden Rollenabstand Resonanz herstellt. Dann wird die Rollendistanz vergrössert und die Reizelektroden mit der Klemme des secundären Condensators verbunden. Zuletzt wird der Rollenabstand allmählich vermindert, bis eine minimale Contraction auftritt, und sogleich $i_{11}$ am Hitzdrahtampèremeter abgelesen. Bei der Ausführung des Versuchs tritt der Umstand, dass meistens ein Tetanus bemerklich wird, bevor das Ampèremeter einen ablesbaren Ausschlag gibt, bindernd entgegen. Hier hätte zwar eine Aichung des Schlittenapparates nach mutuellem Inductionscoëfficienten einen Ausweg schaffen können; indessen bätte ich dann sechs Aichungen machen müssen, da ich ab- 
wechselnd zwei primäre und drei secundäre Rollen verwendete. Ich habe darum nach anderen Methoden gesucht und schliesslich die beiden folgenden Methoden 2 und 3 angewandt; die zweite Methode, welche ausserordentlich genau und zuverlässig ist, wurde nur bei wenigen Versuchen gebraucht und hat hauptsächlich gedient, um die letzte, dritte Methode zu controliren.

2. Wird in den secundären Stromkreis ein inductionsloser Widerstand $r$ eingeschaltet, dann ist die E.-M.-K. an den Enden dieses Widerstandes

$$
E_{11}=i_{11} r
$$

wobei $i_{11}$ wieder die Stromstärke am secundären Condensatorkreis vorstellt. Legen wir die Reizelektroden an clie Enden des Widerstandes $r$ an, so lässt sich diese E.-M.-K. zur Reizung des Nerven oder des Muskels benutzen. Indem wir für $r$ Widerstände von 1 bis $50 \Omega$ benutzen, können wir E.-M.-K. messen von 0,05 bis 15 Volt, da das Ampèremeter von 0,05 bis 0,3 Ampère anzeigt. Nur sollen die Widerstände aus geradlinig ausgespanntem, möglichst dünnem Neusilberdraht bestehen. Der Grund, warum wir diese Methode nicht fortwährend benutzt haben, liegt darin, dass mit dem Einschalten von grösseren Widerständen die Belastung des secundären Stromkreises wächst, was eine kleine Aenderung der Sehwingungsdauer verursacht. Ausserdem wird die Herstellung einer vollkommenen Resonanz immer weniger genau, je grössel der eingeschaltete Widerstand. Bei meinen Versuchen bin ich nur ausnahmsweise über $40 \Omega$ gegangen.

3. Von allen diesen Beschwerden völlig frei ist die dritte Methode. Hierbei wird der secundäre Wechselstrom benutzt als Primärstrom für einen zweiten Lufttransformator in der Form eines sehr k]ejnen, sehr genau aus Fhonit gearbeiteten Inductionsapparates. Den der secundären Rolle dieses zweiten Inductionsapparates entnommenen Strom werden wir als tertiären Strom bezeichnen. - Der tertiäre Stromkreis wird von den. Reizelektroden mit dem Präparat geschlossen. Die in dem tertiären Kreis inducirte elektromotorische Kraft $E_{111}$ wird berechnet aus der Stromstärke $i_{11}$ im secundären Kreis, dem muttiellen Inductionscoëfficienten $\boldsymbol{M}$ des zweiten Inductionsapparates bei der betreffenden Rollendistanz und aus der Frequenz $n$, nach der Formel :

$$
E_{111}=2 \pi n \not M i_{11},
$$

während die an den Reizelelitroden wirksame Klemmenspannung 


$$
e_{111}=\sqrt{\left\{1+\frac{2 \pi n M}{R^{2} n^{2} L^{2}}{ }^{2} \frac{111}{R^{2}}\right\}}
$$

Es zeigte sich recht bald, dass wir statt $e_{111}$ nur $E_{111}$ zu berechnen brauchten, da der Nenner des Bruches im zweiten Gliede der letzteren Formel nur unerheblich von der Einheit verschiedeu ist. $L_{111}$ wurde bestimmt zu 5246 Mikrohenry, also rund $1 / 200$ Henry. Da $R$ immer grösser war als $30000 \Omega$, so betrug bei 250000 Oscillationen der durch die Vernachlässigung des Nenners verursachte Fehler ungefähr $2 \%$, also etwas weniger als der systematische Versuchsfehler, welcher auf etwa $3 \%$ zu veranschlagen ist.

Von den Grössen $i_{11}, n$ und $M$ lässt sich $i_{11}$ sogleich an Hitzdrahtinstrument ablesen. Die Frequenz wird mit der Resonanzmethode bestimmt. Hierbei ist zu beachten, dass jetzt in dem secundären Condensatorkreis die Selbstinduction von zwei Spiralen gebildet wird, nämlich von der secundären Spirale des ersten und von der primären Spule des zweiten Inductionsapparates. Da es sich um in Serie geschaltete Inductionsspulen handelt, ist der resultirende Autoinductionscoëfficient einfach gleich der Summe der einzelnen Inductionscoëfficienten zu setzen. Die primäre Spule des zweiten Inductionsapparates trug zwei Lagen von je 70 Windungen mit einem Selbstinductionscoëfficienten $\lambda=22,1$ Mikrohenry.

$M$, der mutuelle Inductionscoëfficient musste für jede Rollendistanz ermittelt werden. Ich habe dabei zwei verschiedene Methoden ${ }^{1}$ ) angewendet, die vollkommen übereinstimmende Zahlen ergaben für jede um je $1 \mathrm{~cm}$ steigende Distanz von $0-20 \mathrm{~cm}$. Die Resultate wurden in ein Coordinatennetz eingetragen und durch eine stete Curve verbunden. Bei der Berechnung der Versuche habe ich das zu jeder Rollendistanz gehörige $M$ immer dieser Curve entnommen. Die Resultate der Aichung ist in folgender Tabelle niedergelegt, wobei die Coëfficienten in Mikrohenry gegeben sind.

\section{Tabelle I.}

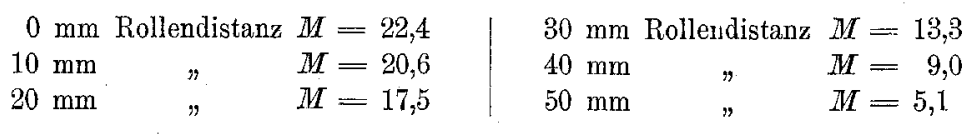

1) Eine Galvanometermethode (v. Kohlrausch, Compendium S. 482, I u. III, und eine Nullmethode Carey Foster's (Phil. Mag. vol. 23 ser. 5 p. 121. 1887), bei der ich ein Telephon benutzt habe. 


$\begin{array}{rlrl}60 \mathrm{~mm} & \text { Rollendistanz } M & =1,83 \\ 70 \mathrm{~mm} & \# & M & =0,55 \\ 80 \mathrm{~mm} & " & M & =0,27 \\ 90 \mathrm{~mm} & " & M & =0,16 \\ 100 \mathrm{~mm} & " & M & =0,10\end{array}$

$\begin{array}{lll}110 \mathrm{~mm} & \text { Rollendistanz } M=0,07 \\ 120 \mathrm{~mm} & \Rightarrow & M=0,05 \\ 130 \mathrm{~mm} & \# & M=0,038 \\ 140 \mathrm{~mm} & \# & M=0,03 \\ 150 \mathrm{~mm} & \Rightarrow & M=0,025\end{array}$

\section{Versuche.}

1. Bogenlampe gespeist mit 2,6 Ampère. Gleichstrom-Klemmenspannung 36,7-37 Volt. Capacität des primären Condensators in maximo 2 Mikrofarad; derselbe wird immer weiter herabgesetzt, bis bei dem letzten Versuche nur noch 0,1 Mikrofarad im primären Condensatorkreis vorhanden waren. Der secundäre Selbstinductionscoëfficient betrug 78,8 Mikrohenry. Vor jeder Reizung wurde genaue Resonanz hergestellt. Die Reizelektroden konnten angelegt werden an den Enden eines geradlinig eingespannten Neusilberdrahts von 10,20 oder $40 \Omega$ Widerstand, welcher in den secundären Stromkreis geschaltet war. Schliesslich wurde die secundäre Spirale so weit über die primäre geschoben, bis eine Contraction auftrat; sogleich wurde die Verbindung mit den Reizelektroden unterbrochen und das Ampèremeter abgelesen. Der ganze Vorgang wurde bei anderen Frequenzen wiederholt.

Tabelle II.

Gastrocnemiuspräparat. Reizung des Nerven.

\begin{tabular}{c|c|c|c|c|c}
\hline \hline$L_{\mathbf{1 1}}$ & $C_{\mathbf{1 1}}$ & $N_{\mathbf{1}}$ & $r$ & $J_{\mathbf{1 1}}$ & $P D$ \\
\hline 78,8 & 0,875 & 19180 & 10 & 0,116 & 1,16 \\
78,8 & 0,47 & 27030 & 20 & 0,114 & 2,28 \\
78,8 & 0,215 & 39960 & 20 & 0,186 & 3,72 \\
78,8 & 0,135 & 50420 & 40 & 0,179 & 7,16 \\
78,8 & 0,075 & 67700 & 40 & 0,230 & 9,2
\end{tabular}

Der Widerstand des Präparates betrug am Ende des Versuchs $61800 \Omega$.

Es bedeutet:

$L_{11}$ den sekundären Selbstinductionscoëfficienten in Mikrohenry, $C_{11}$ Capacität des secundären Condensators in Mikrofarad,

$n$ die aus den beiden vorhergenannten Grössen mittelst der Formel : $n=\frac{10^{6}}{2 \pi \sqrt{L_{11}} C_{11}}$ berechnete Frequenz,

$r$ den Widerstand in Ohm, an dessen Enden die dem Nerven den Strom zuführenden Drähte angelegt waren. 
$J_{11}$ ist die Stromstärke, welche diesen Widerstand durchfloss.

$P D$ ist die Potentialdifferenz, welche an den Enden von $r$ bestand; sie wurde berechnet als $P D=r \times J_{11}$.

2. I dem. Anstellung des Versuchs wie oben. Statt eines ausgeschnittenen Ischiadicus-Gastrocnemius wurde ein Ischiadicus in situ gereizt, nachdem derselbe unter möglichster Schonung auf eine kleine Strecke freipräparirt war. $W$, der Widerstand des Nerven, wurde nicht bestimmt.

Tabelle III.

\begin{tabular}{c|r|r|r|r|r}
\hline$L_{11}$ & $C_{11}$ & $N_{11}$ & $r$ & $J_{11}$ & $P D$ \\
\hline 684,7 & 0,450 & 9080 & 1,8 & 0,168 & 0,301 \\
684,7 & 0,243 & 12350 & $\mathbf{1} 8$ & 0,290 & 0,522 \\
684,7 & 0,074 & 22380 & 10 & 0,105 & 1,05 \\
& & & & &
\end{tabular}

3. Vergleichung der Methode 2 und 3. Aufstellung der Verbindungen nach beigefügter schematischer Zeichnung. Der secundäre

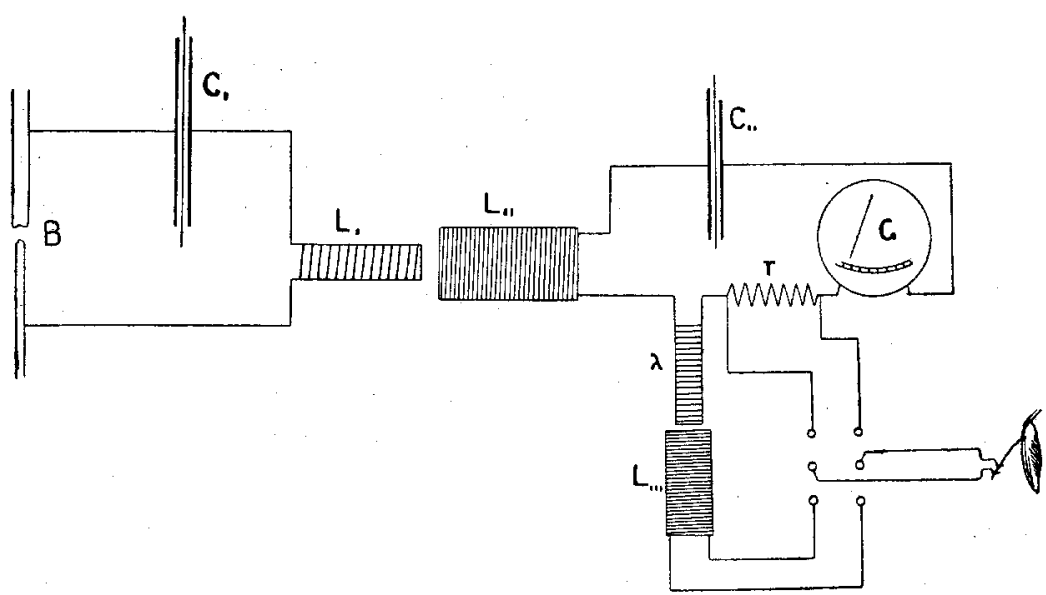

Fig. 3.

Condensatorkreis besteht aus folgenden Theilen: dem secundären Condensator $C_{11}$, der secundären Spirale des ersten Inductionsapparates, dem Hitzdrahtampèremeter $G$, dem Widerstand $r$ von $1,8 \Omega$, der primären Spule $\lambda$ des zweiten Inductionsapparates. - Die Reizelektroden konnten wechselweise entweder an die Klemmen der secundären Spule $L_{111}$ des zweiten Inductionsapparates angelegt 
werden oder auch an die Enden des Widerstandes $r$ von $1,8 \Omega$ (oder $r+G=10 \Omega$ ).

Es wurden zuerst die Reizelektroden an $r$ oder $r+G$ angelegt; dann wurde $L_{11}$ so weit über $L_{1}$ gesehoben, bis eine eben merkliche Contraction auftrat; dann wurde der Commutator umgelegt und $L_{111}$ so weit über $\lambda$ geschoben, bis wieder eine Contraction sichtbar wurde. Die beiden Einstellungen wurden genau controlirt, und zuletzt wurde die Stromstärke abgelesen und die Rollendistanz bei $L_{111}$ notirt. Die Resultate sind in folgender Tabelle niedergelegt.

Tabelle IV.

\begin{tabular}{c|c|c|c|c|c|c|c|c}
\hline$L_{\mathbf{1 1}}$ & $C_{\mathbf{1 1}}$ & $N$ & $r$ & $J_{11}$ & $P D$ & R.-dist. & $M$ & $M K J_{\mathbf{1 1}} \boldsymbol{}$ \\
\hline 706,8 & 0,746 & 6940 & 1,8 & 0,184 & $\mathbf{0 , 3 9 2}$ & 58 & 40,3 & $\mathbf{0 , 3 9 8}$ \\
$\mathbf{7 0 6 , 8}$ & 0,279 & 11390 & 1,8 & 0,278 & $\mathbf{0 , 5 0 2}$ & 59 & 21,5 & $\mathbf{0 , 4 6 9}$ \\
706,8 & 0,158 & 15300 & 10 & 0,078 & $\mathbf{0 , 7 8 0}$ & 37 & 102,2 & $\mathbf{0 , 7 6 6}$
\end{tabular}

Widerstand der Nerven: $44000 \Omega$.

Die ersten sechs Reihen haben wieder die nämliche Bedeutung wie in den letzten Tabellen. R.-dist. heisst Rollendistanz. $M$ ist der zugehörige mutuelle Inductionscoëfficient, während die letzte Reibe die nach der dritten Methode berechnete wirksame Potentialdifferenz enthält. Wir sehen, dass diese Zahlen in befriedigender Weise mit den in der Reihe der $P D$ verzeichneten Zahlen übereinstimmen; während diese mittelst einer absolut strengen Methode erhalten wurde, hängen wir bei der dritten Methode noch selır viel $a b$ von der Genauigkeit, mit der jeder einzelne Coëfficient bestimmt wurde. Obgleich die Uebereinstimmung eine ziemlich gute war, musste es doch auffallen, dass sämmtliche drei nach Methode III erhaltenen Zahlen kleiner waren als die nach Methode II. Ich habe, nachdem diese Versuche berechnet waren, meine sämmtlichen physischen Versuche noch ein Mal wiederholt, wobei ich die nämlichen Resultate erhielt. Bei neuen Vergleichungsversuchen erhielt ich immer einen etwas zu geringen Werth bei der dritten Methode. Es zeigte sich aber, dass bei allen Versuchen der Fehler - der etwa $5-6 \%$ betrug - ziemlich constant war.

So ergab folgende Versuchsreihe bei e in er einzigen Frequenz: 
Tabelle V.

\begin{tabular}{c|c|c|c|c|c|c|c|c}
\hline$L_{11}$ & $C_{11}$ & $N$ & $r$ & $J_{11}$ & $P D$ & $R \cdot-A$. & $M$ & $M J_{11} \omega$ \\
\hline 100,94 & 0,067 & 61200 & 20 & 0,216 & $\mathbf{4}, \mathbf{3 2}$ & 51,0 & 47,0 & $\mathbf{3 , 9 1}$ \\
100,94 & 0,067 & 61200 & 20 & 0,210 & $\mathbf{4 , 2 0}$ & 50,0 & 51,0 & $\mathbf{4 , 1 2}$ \\
100,94 & 0,067 & 61200 & 20 & 0,217 & $\mathbf{4 , 3 4}$ & $\mathbf{5 0 , 5}$ & 49,1 & $\mathbf{4 , 0 8}$
\end{tabular}

Der Mittelwerth für $P D$ beträgt 4,29; derjenige für $M J_{11}(\omega, 04$, d. h. sie zeigen einen Unterschied von $6^{\circ} \%$.

Der Unterschied scheint constant zu sein. Wir dürfen also annehmen, dass sämmtliche Zahlen, die nach der dritten Methode erhalten wurden, um etwa $6 \%$ zu niedrig sind.

Nach diesen Vorversuchen habe ich getrachtet einige Reihen zu erhalten, die sich über ein grösseres Gebiet ausdehnten. Das Resultat dieser Versuchsreihen ist in folgenden Tabellen niedergelegt.

Tabelle VI.

\begin{tabular}{l|l|r|r|r|l|l}
\hline \hline$I \cdot 10^{-6}$ & $C_{2} \cdot 10^{-6}$ & $N$ & $R \cdot \cdot A$ & $M \cdot 10^{-6}$ & $J$ & $M \omega J$ \\
& & & & & \\
\hline 100,94 & 0,5 & 22390 & 27,5 & 59,08 & 0,228 & 1,89 \\
100,94 & 0,069 & 60310 & 8,0 & 85,3 & 0,20 & 6,46 \\
100,94 & 0,062 & 63620 & 13,5 & 79,8 & 0,21 & 6,70 \\
100,94 & 0,092 & 52.230 & 25,0 & 62,0 & 0,26 & 5,31 \\
100,94 & 0,020 & 112000 & 22,0 & 67,2 & 0,23 & 10,91 \\
100,94 & 0,024 & 102200 & 19,0 & 72,2 & 0,24 & 10,21 \\
100,94 & 0,004 & 250400 & 30,0 & 53,2 & 0,21 & 17,56 \\
100,94 & 0,190 & 36340 & 27,0 & 59,5 & 0,225 & 3,06 \\
2720 & 0,037 & 15870 & 33,2 & 48,0 & 0,25 & 1,05 \\
2720 & 0,075 & 11140 & 44,0 & 30,2 & 0,22 & 0,550 \\
2720 & 0,093 & 10006 & 44,2 & 29,6 & 0,26 & 0,485 \\
2720 & 0,100 & 9649 & 44,0 & 30,2 & 0,26 & 0,476 \\
2720 & 0,184 & 7114 & 49,0 & 21,6 & 0,25 & 0,241 \\
2720 & 0,304 & 5535 & 47,0 & 24,2 & 0,215 & 0,181 \\
100,94 & 0,004 & 250400 & 34,5 & 46,3 & 0,21 & 15,63 \\
100,94 & 0,004 & 250400 & 37,0 & 40,6 & 0,28 & 17,85 \\
& & & & & &
\end{tabular}

$$
\mathrm{W}=42200 \Omega \text {. }
$$

Zu Tabelle VII ist zu bemerken, dass die niedrigsten Frequenzen nur erhalten werden konnten, indem in den primären Condensatorkreis zwei grosse Drahtspulen mit etwa 150 Windungen von $30 \mathrm{~cm}$ Durchmesser eingeschaltet wurden. Die Capacität daselbst wurde erhöht bis auf 3,6 Mikrofarad. Zur Messung dieser niedrigen Frequenzen, 2450-6000 pro Secunde, musste auch in dem secundären 
Condensatorkreis die Selbstinduction erhöht werden: es geschah dies in einfacher Weise mittelst Einschaltung einer kleinen secundären Spirale mit einer Selbstinduction von 5246 Mikro-Henry: die gesammte Selbstinduction des secundären Condensatorkreises betrug dabei 7966 Mikro-Henry. Die Gleichstromspannung am Lichtbogeu betrug 42 Volt, wurde aber erniedrigt bis auf 38 Volt bei den weiteren Versuchen.

Tabelle VII.

\begin{tabular}{|c|c|c|c|c|c|c|}
\hline$L \cdot 10^{-6}$ & $C_{2} \cdot 10^{-6}$ & $N$ & $R .-A$ & $M \cdot 10^{-6}$ & $J$ & $M_{\omega} J$ \\
\hline 7966 & 0,53 & 2449 & 51 & 18,9 & 0,223 & 0,0648 \\
\hline 7966 & 0,44 & 2688 & 50 & 20,3 & 0,235 & 0,0805 \\
\hline 2720 & 0,24 & 6229 & 48 & 23,2 & 0,23 & 0,209 \\
\hline 2720 & 0,204 & 6756 & 44,3 & 29,3 & 0,185 & 0,230 \\
\hline 2720 & 0,17 & 7401 & 45 & 28,8 & 0,20 & 0,268 \\
\hline 2720 & 0,1 & 9649 & 46 & 27,2 & 0,25 & 0,412 \\
\hline 2720 & 0,047 & 15790 & 38 & 39,2 & 0,205 & 0,796 \\
\hline 100,94 & 0,54 & 21560 & 33 & 49,2 & 0,207 & 1,380 \\
\hline 100,94 & 0,38 & 27590 & 35 & 44,3 & 0,26 & 1,996 \\
\hline 100,94 & 0,077 & 57110 & 29 & 56,1 & $(1,25$ & 5,033 \\
\hline 100,94 & 0,022 & 106800 & 14 & 79,3 & 0,20 & 10,90 \\
\hline 100,94 & 0,021 & 109300 & 11 & $8 \%, 6$ & 0,21 & 11,64 \\
\hline 100,94 & 0,012 & 144600 & 8 & 85,3 & 0,20 & 15,50 \\
\hline 100,94 & 0,007 & 189400 & 16 & 76,8 & 0,19 & 17,37 \\
\hline
\end{tabular}

$W=49800 \Omega$.

Tabelle VIII.

\begin{tabular}{l|l|r|r|r|l|l}
\hline \hline$L \cdot 10^{-6}$ & $C \cdot 10^{-6}$ & $N$ & $R \cdot-A$. & $M \cdot 10^{-6}$ & $J$ & $2 \pi N M J$ \\
& & & & & & \\
\hline & & & & & & \\
2720 & 0,1 & 9650 & 61,5 & 5,8 & 0,28 & 0,099 \\
2720 & 0,054 & 13280 & 58,5 & 9,3 & 0,247 & 0,192 \\
2720 & 0,021 & 21090 & 49 & 22,1 & 0,24 & 0,703 \\
2720 & 0,009 & 32170 & 42 & 33,4 & 0,195 & 1,32 \\
100,94 & 0,13 & 43930 & 39 & 37,7 & 0,23 & 2,39 \\
100,94 & 0,017 & 121500 & 29 & 56,6 & 0,25 & 10,80 \\
100,94 & 0,004 & 250500 & 31 & 52.8 & 0,20 & 16,46 \\
100,94 & 0,08 & 56000 & 35 & 45,0 & 0,24 & 3,80 \\
100,94 & 0,01 & 158400 & 31 & 52,3 & 0,27 & 14,05 \\
100,94 & 0,34 & 27160 & 45,5 & 27,4 & 0,215 & 1,006 \\
100,94 & 0,037 & 82350 & 31 & 52,3 & 0,24 & 6,50 \\
100,94 & 0,27 & 30480 & 45 & 28,5 & 0,22 & 1,20 \\
100,94 & 0,80 & 16710 & 56 & 12,9 & 0,26 & 0,373 \\
100,94 & 0,09 & 52800 & 38 & 39,6 & 0,24 & 3,15 \\
& \multicolumn{7}{|c|}{$W=61000 \Omega}$. & &
\end{tabular}

Ich habe diese Tabellen gegeben in der Reihenfolge der Einzelversuche, wie dieselben ausgeführt wurden. 
Um einen besseren Ueberblick über die erhaltenen Resultate zu gewinnen, habe ich dieselbe in beigehender Fig. 4 zusammengestellt. Dabei sind die Logarithmen der Frequenzen als Ordinaten, die Logarithmen der Potentialdifferenzen als Abscissen benutzt. Der

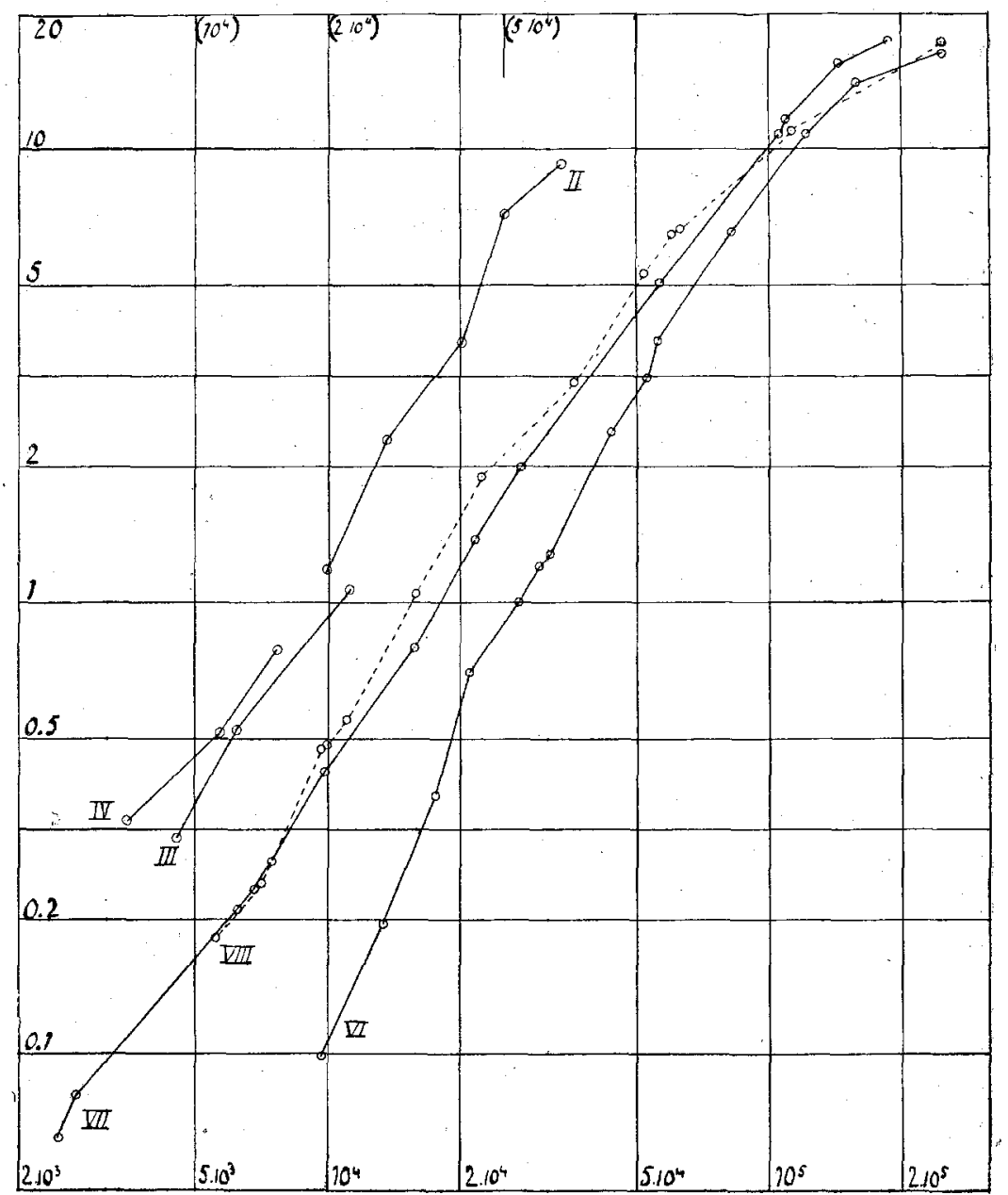

Fig. 4 .

charakteristische Verlauf der Curven wird dadurch nicht beeinträchtigt, nur sind die unteren 'Theile der Curven einigermassen auseinandergezogen, während die höheren Theile zusammengedrängt sind. Ausserdem ist zu bemerken, dass die Curven für die Tabellen II, III und IV in toto über eine Ordinatendistanz von log 2 nach links verschoben sind, und dass die an der oberen Seite der Figur in Klammern 
gestellten Zahlen auf diese drei Curven zu beziehen sind, während die Zahlen an der unteren Seite der Figur auf die längeren Curven VI, VII und VIII Beziehung haben. Jede Curve ist bezeichnet mit der Nummer der zugehörigen Tabelle.

Ein Blick auf die Fig. 4 zeigt uns sogleich, dass die sämmtlichen Curven einen sehr gestreckten, fast geradlinigen Verlauf baben, jedoch mit einer unverkennbaren Neigung zur Krümmung in der Richtung zur X-Achse, der sie also ihre Concavität zuwenden, und namentlich am oberen Theil der Curve.

Die Erregbarkeit von sämmtlichen untersuehten Nerven war offenbar wenig verschieden, und nur der Nerv der Tabelle VI zeigt eine etwas grössere Erregbarkeit. Sämmtliche Versuche fanden statt im October und November 1903.

Ueber das Anwachsen der Stromstärke, die gerade zum Hervorrufen eines Tetanus genügt, bestehen bis jetzt drei verschiedene Theorien, die zu drei verschiedenen Formeln führten.

$\alpha$. Hoorweg ${ }^{1}$ ) leitet aus seinem Reizgesetz eine Formel ab, die in möglichst einfacher Gestalt dargestellt wird als:

$$
J=k_{1} n+k_{11} \frac{1}{n}
$$

wobei $d$ die benöthigte Stromstärke, $n$ die Frequenz und $k_{1}$ und $k_{11}$ zwei Constanten sind.

Diese Formel stellt eine gebogene Linie vor mit einem Minimum und einem endlichen Werth für $n=0$. Bei sehr grossem $n$ geht dieselbe über in eine gerade Linie. Die Versuchsresultate von v. Kries schliessen sich sehr genau an diese Formel an. Meine eignen Resultate stehen nicht in directem Widerspruch mit dieser Formel, obgleich das relative Herabsinken der Stromstärke bei Frequenzen über 100000 nicht erklärt wird. Hierfür besteht jedoch wahrscheinlich eine einfache physische Ursache (s. u.).

ß. Her nann ${ }^{2}$ ) gibt die Formel:

1) Hoorweg, Ueber Nervenerregung durch sogenannte Wechselströme. Pflüger's Arch. Bd. 83 S. 89-98. - Ueber die Erregung der Nerven. Pflüger's Arch. Bd. 85 S. 106. - Ueber die Erregung der Nerven. Pflüger's Arch. Bd. 87 S. 94. - Recherches sur l'excitation électrique des nerfs. Arch. de Teyler, Série III t. 7 cinq. part p. 70-97. 1901.

2) Hermann, Ceber Nervenerregung durch Wechselströme und die Theorie der Nervenerregung. Pflüger's Arch. Bd. 83 S. 356. 


$$
J=\text { const. } \frac{\left.\sqrt{m^{2} w^{2}+\left(h-m^{2}\right.} \bar{k}\right)^{2}}{m^{2}},
$$

wobei $m=2 \pi n$.

Nehmen wir diese Formel:

$$
d=\text { const. } \sqrt{\frac{w^{2}}{m^{2}}+\left(\frac{h^{2}}{m^{2}}-k\right)^{2}},
$$

dann sehen wir sogleich, dass bei genügender Vergrösserung von $m$ die Stromstärke $J$ sich asymptotisch einem constanten Werth nähert und praktisch bei grossem $m$ unabhängig von der Frequenz ist. Mit dieser Formel stehen unsere Resultate in directem Widerspruch.

$\gamma$. Nernst ${ }^{1}$ ) gab schliesslich eine Theorie, welche zu der Formel führt:

$$
J=k \sqrt{n}
$$

Bei steigender Frequenz steigt auch die Stromstärke, und zwar proportional der Quadratwurzel der Frequenz. Wie elegant die Nernst'sche Theorie auch sein mag, muss ich doch betonen, dass dieselbe den Thatsachen absolut nicht entspricht. Eine kleine Rechnung für meine Versuchsresultate zeigt, dass sich diese der Nernst'schen Formel nicht anpassen lassen. Ich muss hier auch betonen, dass die v. Kries'schen Versuchsergebnisse sich ebenfalls der Nernst'schen Formel nicht fügen. Selbst die neuerdings von Nernst ${ }^{2}$ ) und Barratt veröffentlichten Versuche müssen als wenig geeignet bezeichnet werden, um seine Theorie zu stützen. Wer sich die Mühe gibt, seine drei Reihen graphisch aufzutragen, gewinnt sogleich den Eindruck, dass für dieselben ein einfach lineares Gesetz fast gerade so gut passen würde. Thatsächlich ergiebt die Rechnung für seine erste Reihe eine Verminderung der Summe der Fehlerquadrate auf etwa die Hälfte (von 0,0193 auf 0,0103), wenn dieselbe statt nach dem Quadratwurzelgesetz nach einem lineären Gesetze berechnet werden.

Seine zweite Tabelle ergiebt eine etwas grössere Abweichung vom einfachen lineären Gesetz, aber eine bessere Uebereinstimmung mit Hoorweg's Formel, mit der auch seine letzte Reihe ziemlich gut übereinstimmt.

1) Nernst, Gött. Nachr., math.-physik. Classe 1899 H. 1 S. 104.

2) Nernst und Barratt, Ueber die elektrische Nervenreizung durch Wechselströme. Kurella's Zeitschr. f. Elektrotherapie Bd. 6 H. 8 S. 253. 
Die zur Erregung des Nerven erforderliche Stromstärke wächst bei Erhöhung der Frequenz von 2500 bis auf 250000 pro Secunde sehr bedeutend an. Die Stromstärke erhöht sich dabei von $0,0648: 49800=1,3$ Mikroampère auf $17,85: 49800=358$ Mikroampère oder 0,358 Milliampère. In dem vom Strome durchflossenen Theil des Nerven wird dabei eine Energie von $i^{2} R=\overline{0,358}^{2} \cdot 10^{-6}$. $49800=0,006$ Watt verbraucht, wobei dem Nerven in jeder Secunde eine Quantität Wärme zugeführt wird von $0,006 \times 0,239$ WasserGramm-Kal./sec. $=0,001434$. Wenn das Gewicht des $10 \mathrm{~mm}$ langen, vom Strome durchflossenen Theils des Nerven auf etwa $35 \mathrm{mg}$ veranschlagt wird, und seine specifische Wärme gleich der des Wassers gesetzt wird, so wird die Temperatur eines Nerven von einem Wechselstrom von 250000 Perioden in jeder Secunde um etwa $1,434: 35=0,04^{0}$ erhöht werden. Ein Durchfliessen während 25 Secunden erhöht die Temperatur schon um $1^{0}$. Da diese Temperaturänderung eine erhebliche Aenderung der Erregbarkeit verursacbt, und zwar im Allgemeinen eine Erhöhung derselben, so dürfte sich hierindie Erklärung finden für die eigenthümliche Krümmung der Curven in ihren oberen Theilen. Könnten wir die Temperatur absolut constant halten, dann würde der Verlauf wahrscheinlich gänzlich geradlinig sein und im grossen Ganzen Hoorweg's Formel entsprechen. Ich habe kein Bedenken, in meinen Versuchsergebnissen eine kräftige Stütze für die Richtigkeit der Hoorweg'schen Formel zu sehen. Jedenfalls geht aus meinen Versuchen mit Bestimmtheit hervor, dass bei zunehmender Frequenz der Wechselströme die zur minimalen Erregung eines Nerven benöthigte Intensität nach einem lineären Gesetz zunimmt zwischen den Grenzen 2000-100000; über 100000 ist die Zunahme etwas geringer, als mit einem lineären Verhältniss erforderlich ist; dieses Zurückbleiben dürfte jedoch in einfacbster Weise erklärt werden durch die Temperaturerhöhung und die damit einhergehende erhöhte Erregbarkeit in Folge des Durchfliessens des Stromes. 